\title{
AMBULATORY MICRO-DOSE INDUCTION OF BUPRENORPHINE-NALOXONE IN TWO ADOLESCENT PATIENTS WITH SICKLE CELL DISEASE
}

\author{
Bradley Buchheit ${ }^{1}$, Timothy Joslin ${ }^{1}$, Helen Turner ${ }^{1}$, and Trisha E. Wong ${ }^{1}$ \\ ${ }^{1}$ Oregon Health \& Science University
}

June 15, 2020

\begin{abstract}
Sickle cell disease (SCD) is a hematologic disorder defined by presence of sickle-shaped red blood cells that can occlude blood vessels and cause tissue ischemia and pain. Treating SCD pain adequately and safely is difficult given today's opioid climate. Buprenorphine-naloxone is described in adult literature where it typically requires withdrawal of full-agonist opioids and inpatient hospitalization. Here we present two adolescents with SCD who successfully weaned off large doses of full-agonist opioids by using micro-dose induction of buprenorphine-naloxone in clinic. Ambulatory micro-dose induction may remove hurdles that otherwise would discourage patients from trying this regimen while still controlling pain safely.
\end{abstract}

\section{Introduction}

Sickle cell disease (SCD) is a hematologic disorder defined by presence of a mutation in the $H B B$ gene that results in sickle-shaped red blood cells (RBC). These misshapen, rigid RBCs can occlude blood vessels causing tissue ischemia and intense pain. Although this vaso-occlusive pain best characterizes SCD pain, many patients have more complex pain manifestations including chronic, persistent pain. While opioids are the mainstay of treatment for pain in SCD, patients may become physically and emotionally dependent on opioids; with some developing opioid use disorder (OUD).

Buprenorphine is a mixed agonist-antagonist agent that exerts analgesic effects by binding to CNS opioid receptors. Buprenorphine-naloxone has been used to treat OUD since 2002. ${ }^{1}$ Buprenorphine has a high affinity to mu-opioid receptors, which can result in precipitated opioid withdrawal symptoms if given too soon after a full opioid agonist in opioid-dependent individuals. Historically, induction of buprenorphine in opioiddependent individuals required mild to moderate opioid withdrawal and cessation of full opioid agonists for 24-48 hours. ${ }^{2}$ Case reports describe alternative approaches utilizing small, frequent doses of buprenorphinenaloxone to avoid withdrawal symptoms and rapidly transition patients off full agonist opioids. ${ }^{3-12}$ None of these case studies include adolescents or individuals with SCD.

Here we describe two adolescent patients with SCD who were safely transitioned to buprenorphine-naloxone using a micro-dose induction protocol (Bernese Method) ${ }^{11,12}$ in an outpatient setting.

Case 1

Patient 1 is a 16 -year-old male with Hemoglobin SC disease with no SCD complications prior to 2016. In 2016, he presented 13 times to local emergency departments (ED) for acute pain. Oxycodone immediaterelease (IR) was prescribed to treat and prevent escalation of pain. In 2017, he presented to the ED 17 times and was hospitalized for 36 days leading to the addition of oxycodone extended-release (ER). On this regimen, his ED visits decreased, he did not require hospitalization, and he returned to school and extra-curricular activities. 
He continued to do well, and in early 2019, oxycodone IR and ER were slowly tapered. This triggered multiple presentations to the ED for pain, where SCD and non-SCD etiologies were ruled out, and he was discharged to continue the oxycodone taper. Tapering efforts continued to be unsuccessful and he was transitioned from oxycodone ER to methadone, while increasing his oxycodone IR. Unfortunately, his high ED utilization continued, expanded to other hospital systems, and he faced discontinuation of his opioids due to controlled substance agreement violations.

In December 2019, the patient's primary care was transitioned from a community pediatrician to a family medicine physician, co-boarded in addiction medicine. His daily regimen was methadone $12.5 \mathrm{mg}$ and oxycodone IR $37.5 \mathrm{mg}$ (approximately 106 morphine milligram equivalents (MME)). At this time, the patient did not meet criteria for an opioid use disorder, but showed signs of opioid dependence and risky use. The patient's goal was to wean off opioids. The patient and his PCP discussed initiating buprenorphine-naloxone for persistent pain given its favorable side effect profile and long-acting nature. He was amenable, and in late December 2019 buprenorphine-naloxone was initiated using the Bernese Method of micro-dose induction, Table $1.11,12$

The patient denied opioid withdrawal symptoms or side effects. He reported two migraines during induction, which were responsive to his standard migraine medications. The patient has been on buprenorphine for five months and is currently taking $1 \mathrm{mg}-0.25 \mathrm{mg}$ SL daily with the plan to discontinue the medication completely in two weeks. He reports improved pain control, has not utilized any ED or hospital services, and has not missed any school since induction. During visits with pediatric hematology and his PCP, he consistently reports feeling like a normal teenager, and plans to return to playing soccer.

Case 2

Patient 2 is 19-year-old male with Hemoglobin SC disease complicated by acute vascular necrosis of bilateral hips at age 7 and a one-week course of extracorporeal membrane oxygenation (ECMO) in June 2018 for multisystem organ failure due to a presumed fat embolus. He has a history of acute and persistent pain since early childhood and was taking up to 330 MME per day of oxycodone while finishing high school and working part-time. For transition to an adult primary care provider at age 18, his hematology team sought a provider with experience managing patients with persistent pain.

Upon transitioning to a family medicine PCP, intermittent urine drug screens and regular checks of the Prescription Drug Monitoring Program showed no evidence of diversion. In preparation for college, in January 2020, he requested his opioid regimen be tapered and discontinued. After multiple discussions, and despite a slow and controlled taper, he experienced withdrawal symptoms (body aches, worsening pains, sweats) that he attributed to SCD which halted the taper and led to return to previous oxycodone doses. During this time, due to multiple admissions for pain crises, his total opioid dose actually increased.

The idea of rapid, opioid taper using a buprenorphine-naloxone micro-dose induction was discussed over several visits with the patient. Prior to undergoing the micro-dose induction, the patient was taking oxycodone ER 40mg three times daily and 60mg oxycodone IR daily (270 MME). In February 2020, using the micro-dose induction protocol, the patient successfully transitioned to buprenorphine-naloxone, Table 2. He reported only sweats during the micro-induction. After induction, he reported yawning, chills and sweats relieved by taking additional buprenorphine-naloxone as needed. His sweating resolved with changing from the tablet to the film formulation. The patient's current dose is $4 \mathrm{mg}-1 \mathrm{mg}$ twice daily. In the months since his micro-induction, he has had brief episodes of pain managed with small doses of oxycodone IR and administration of IV opioids in the hematology clinic. He has avoided admission for pain since transitioning to buprenorphine-naloxone, compared to the year prior in which he had six hospital admissions (total 56 days).

\section{Discussion}

These cases highlight the benefits of micro-dose induction versus conventional induction: it can make buprenorphine-naloxone more enticing for patients who do not want to withdraw from opioids, reduces hospital utilization because it is done outpatient, and preserves the benefits of conventional induction. Fur- 
thermore, in patients with SCD on chronic opioids, where a conventional induction could trigger a sickle cell pain episode due to the biologic and emotional stress of opioid withdrawal, micro-induction may have added benefits.

Limitations of this case series is that only two individuals were included, both of whom have Hemoglobin SC disease, and not the more common and typically more severe Hemoglobin SS disease. However, effectiveness and safety should not differ substantially between the two genotypes. More research is needed on important outcomes, such as pain management, healthcare utilization, quality of life, and cost-effectiveness.

\section{CONFLICT OF INTEREST STATEMENT}

The authors declare no potential conflict of interest.

\section{AUTHOR CONTRIBUTIONS}

$\mathrm{BB}$, TJ, and TEW contributed to clinical care and primary manuscript composition. HT contributed to clinical care and made critical edits.

\section{REFERENCES:}

1. Drug Enforcement Agency - Drug \& Chemical Evaluation Section.Buprenorphine (Trade Names: Buprenex $($, Suboxone $($, Subutex(R) .; 2019.

2. SAMHSA. Treatment Improvement Protocol (TIP) 63 - Medications for Opioid Use Disorder .; 2020. https://store.samhsa.gov/product/TIP-63-Medications-for-Opioid-Use-Disorder-Full-Document/PEP20-0201-006.

3. Terasaki D, Smith C, Calcaterra SL. Transitioning Hospitalized Patients with Opioid Use Disorder from Methadone to Buprenorphine without a Period of Opioid Abstinence Using a Microdosing Protocol.Pharmacotherapy . 2019;39(10):1023-1029. doi:10.1002/phar.2313

4. Sandhu R, Zivanovic R, Klaire S, Nikoo M, Rozylo J, Azar P. Buprenorphine/naloxone induction for treatment of acute on chronic pain using a micro-dosing regimen: A case report. Can J Pain . 2019;3(1):7984. doi:10.1080/24740527.2019.1599279

5. Rozylo J, Mitchell K, Nikoo M, et al. Case report: Successful induction of buprenorphine/naloxone using a microdosing schedule and assertive outreach. Addict Sci Clin Pract . 2020;15(2). doi:10.1186/s13722-020$0177-\mathrm{x}$

6. Randhawa PA, Brar R, Nolan S. Buprenorphine-naloxone "microdosing": An alternative induction approach for the treatment of opioid use disorder in the wake of North America's increasingly potent illicit drug market. CMAJ . 2020;192:E73. doi:10.1503/cmaj.74018

7. Raheemullah A, Lembke A. Buprenorphine Induction Without Opioid Withdrawal: A Case Series of 15 Opioid-Dependent Inpatients Induced on Buprenorphine Using Microdoses of Transdermal Buprenorphine. Am J Ther . 2019. doi:10.1097/MJT.0000000000001108

8. Raheemullah A, Lembke A. Initiating Opioid Agonist Treatment for Opioid Use Disorder in the Inpatient Setting: A Teachable Moment.JAMA Intern Med . 2019;179(3):427-428.

9. Lee DS, Hann JE, Klaire SS, Nikoo M, Negraeff MD, Rezazadeh-Azar P. Rapid Induction of Buprenorphine/Naloxone for Chronic Pain Using a Microdosing Regimen: A Case Report. A A Pract . 2020;14(2):44-47. doi:10.1213/xaa.0000000000001138

10. De Aquino JP, Fairgrieve C, Klaire S, Garcia-Vassallo G. Rapid Transition From Methadone to Buprenorphine Utilizing a Micro-dosing Protocol in the Outpatient Veteran Affairs Setting. J Addict Med . 2020;00(00). doi:10.1097/adm.0000000000000618 
11. Hämmig R, Vogel M, Kemter A, et al. Use of microdoses for induction of buprenorphine treatment with overlapping full opioid agonist use: the Bernese method. Subst Abuse Rehabil . 2016;Volume 7:99-105. doi:10.2147/sar.s109919

12. Hammig R. Einleitung einer Substitutionsbehandlung mit Buprenorphin unter vorübergehender Überlappung mit Heroinkonsum: ein neuer Ansatz ("Berner Methode") [Induction of a buprenorphine substitution treatment with temporary overlap of heroin use: a new approach ("B.Suchttherapie . 2010;11(3):129132. doi:10.1055/s-0030-1261914

Table 1. Buprenorphine-naloxone outpatient micro-dosing protocol - Case 1

Table 2. Buprenorphine-naloxone outpatient micro-dosing protocol - Case 2

\section{Hosted file}

Amb bup-nal in two adolescent SCD patients_Table 1 (SUBMITTED).docx available at https: //authorea.com/users/333484/articles/459705-ambulatory-micro-dose-induction-ofbuprenorphine-naloxone-in-two-adolescent-patients-with-sickle-cell-disease

\section{Hosted file}

Amb bup-nal in two adolescent SCD patients_Table 2 (SUBMITTED).docx available at https: //authorea.com/users/333484/articles/459705-ambulatory-micro-dose-induction-ofbuprenorphine-naloxone-in-two-adolescent-patients-with-sickle-cell-disease 